\title{
BOUNDARY ELEMENT ANALYSIS OF THIN STRUCTURAL PROBLEMS IN 2D ELASTOSTATICS
}

\author{
Yao-Ming Zhang \\ Institute of Applied Mathematics, Shandong University of Technology, Zibo, Shandong Province, China. Industrial \\ Equipment State Key Laborating of Structures Analysis, Dalian University of Technology, Dalian 116024, China., \\ zymfc@163.com \\ Yan $\mathrm{Gu}$ \\ Institute of Applied Mathematics, Shandong University of Technology, Zibo, Shandong Province, China. \\ Jeng-Tzong Chen \\ Department of Harbor and River Engineering, National Taiwan Ocean University, Keelung 20224, Taiwan.
}

Follow this and additional works at: https://jmstt.ntou.edu.tw/journal

Part of the Engineering Commons

\section{Recommended Citation}

Zhang, Yao-Ming; Gu, Yan; and Chen, Jeng-Tzong (2011) "BOUNDARY ELEMENT ANALYSIS OF THIN STRUCTURAL PROBLEMS IN 2D ELASTOSTATICS," Journal of Marine Science and Technology. Vol. 19: Iss. 4, Article 10.

DOI: $10.51400 / 2709-6998.2182$

Available at: https://jmstt.ntou.edu.tw/journal/vol19/iss4/10

This Research Article is brought to you for free and open access by Journal of Marine Science and Technology. It has been accepted for inclusion in Journal of Marine Science and Technology by an authorized editor of Journal of Marine Science and Technology. 


\section{BOUNDARY ELEMENT ANALYSIS OF THIN STRUCTURAL PROBLEMS IN 2D ELASTOSTATICS}

\section{Acknowledgements}

The research is supported by the National Natural Science Foundation of China (10571110), the Opening Fund of the State Key Laboratory of Structural Analysis for Industrial Equipment (GZ1017) and the National Natural Science Foundation of Shangdong Province of China (ZR2010AZ003). 


\title{
BOUNDARY ELEMENT ANALYSIS OF THIN STRUCTURAL PROBLEMS IN 2D ELASTOSTATICS
}

\author{
Yao-Ming Zhang*,**, Yan $\mathrm{Gu}^{*}$, and Jeng-Tzong Chen***
}

Key words: boundary element method, nearly singular integrals, thin structural problems, elasticity.

\begin{abstract}
Thin structures, such as thin films and coatings, have been widely designed and utilized in many industries recently. However, the widespread experimental research in thin structural problems underlies a general lack of modeling efforts which can accurately and efficiently predict their performance. In this paper, the boundary element method (BEM) based on elasticity theory is developed for two-dimensional (2D) thin structures with the thickness to length ratio in the micro $\left(10^{-6}\right)$ or nano $\left(10^{-9}\right)$ scales. The BEM-based approach proposed in this paper is constructed using a combination of the regularized indirect boundary integral equations (BIEs) and a general nonlinear transformation which can eliminate the nearly singular properties of the integral kernels. For the test problems studied, very promising results are obtained when the thickness to length ratio is in the orders between $10^{-6}$ and $10^{-9}$, which is sufficient for modeling most thin structures in the micro- or nano-scales.
\end{abstract}

\section{INTRODUCTION}

Structures made from plate and shell assemblies, also known as thin-walled structures, are important in many areas of engineering. The efficient solution of such structures has wide application, from the design of more conventional civil engineering systems to more sophisticated structures employed, for instance, by the aircraft industry. However, the widespread experimental research in thin structures underlies a general lack of modeling efforts which can accurately and

Paper submitted 02/05/10; revised 05/04/10; accepted 05/08/10. Author for correspondence: Yao-Ming Zhang (e-mail:zymfc@163.com).

*Institute of Applied Mathematics, Shandong University of Technology, Zibo, Shandong Province, China.

**Industrial Equipment State Key Laborating of Structures Analysis, Dalian University of Technology, Dalian 116024, China.

***Department of Harbor and River Engineering, National Taiwan Ocean University, Keelung 20224, Taiwan. efficiently predict their performance.

For computational models of thin structures or thin shapes in structures, two numerical methods can be employed, namely, the finite element method (FEM) and the boundary element method (BEM). In the last three decades, the FEM based on plate and shell theories has been a successful tool for the analysis of 3-D thin structures such as plates, shells and layered composite structures to study their deformation and stress in macro-scale. However, the FEM element count increases dramatically for thin structures due to aspect ratio limitations, and it was found out that, as shown in Ref. [14], the number of the finite elements were so large that the task quickly exceed the capacity of the computer used as the thickness decreases. The BEM is a powerful and efficient computational method if integrals are evaluated accurately, and the BEM based on the elasticity theory is in general more accurate in stress analysis of structures. This accuracy will be maintained in the analysis of thin structures as well, if the BEM is implemented correctly to deal with the difficulties associated with thin structures.

Studies show that the conventional boundary element method (CBEM) using the standard Gaussian quadrature fails to yield reliable results for thin-walled structures. The major reason for this failure is that the kernels' integration presents various orders of near singularities, owing to the mesh on one side of the thin-body being too close to the mesh on the opposite side. Nearly singular integrals are not singular in the sense of mathematics. However, from the point of view of numerical integrations, these integrals can not be calculated accurately by using the conventional numerical quadrature since the integrand oscillates very fiercely within the integration interval. Other than the nearly singular integral, many direct and indirect algorithms for singular integral have been developed and used successfully $[1-4,6,7,10,11,19,23,24]$. Therefore, the key point in achieving the required accuracy and efficiency of the BEM is not the singular integral but the nearly singular integral. Although that difficulty can be overcome by using very fine meshes, the process requires too much preprocessing and $\mathrm{CPU}$ time.

In the past decades, tremendous effort is devoted to derive convenient integral forms or sophisticated computational techniques for calculating the nearly singular integrals. These 
proposed methods can be divided on the whole into two categories: "indirect algorithms" and "direct algorithms". The indirect algorithms, which benefit from the regularization ideas and techniques for the singular integrals, are mainly to calculate indirectly or avoid calculating the nearly singular integrals by establishing new regularized BIE [5, 13, 17, 20, 22, 27]. The direct algorithms are calculating the nearly singular integrals directly, such as interval subdivision method [9], special Gaussian quadrature method [15], exact integration method $[18,28]$, and various nonlinear transformation methods $[8,16$, $25,26]$. In a recent study, the above methods have been reviewed in detail by Zhang and Sun [26].

With the development of the numerical techniques for calculation nearly singular integrals, considerable progress has been made in the application of the BEM to the analysis of thin walled structures in the past few years. Sladek et al. have obtained amount of the original results in this field [21]. Nonsingular integral equations for thin-walled structures are proposed based on a subtraction technique and mathematical regularization. Liu et al. $[12,14]$ have undertaken a lot of researches in this field. The nearly singular surface integrals are transformed into a sum of weakly singular integrals, and a nonlinear coordinate transformation is developed for nearly weakly singular integrals. The theory is also applied to interfacial stress analysis for multi-coating systems, thermal stress analysis of multi-layer thin films and coatings, and also thin piezoelectric solids. However, in Liu's work only some boundary unknowns can be computed, for example, this approach only gives the results of radial stresses on the boundary nodes. The tangential stresses on the boundary and the physical quantities at interior points need further investigation. Zhou et al. proposed semi-analytical or analytical integral algorithms to solve 2-D or 3-D thin body problems [30, 31]. Both boundary and interior unknowns are computed in their works which the geometry boundary is depicted by using linear shape functions when nearly singular integrals need to be calculated.

Although great progresses have been achieved of each of the above works, it should be point out that most of these earlier methods are neither general used nor providing accurate results when the thickness of the thin structure is smaller than 1.0E-6. The objective of this paper is to develop a general BEM-based simulation for predicting the physical quantities in thin structures when the thickness to length ratio is in the orders of $1.0 \mathrm{E}-6$ to $1.0 \mathrm{E}-9$.

It is well-known that the geometries of many problems of practical interest are created from circular or elliptic arcs. Arc boundary elements can represent circular and elliptic boundaries exactly, and consequently, errors caused by representing such geometries using polynomial shape functions can be removed using exact geometrical representations. Therefore, the exact geometrical representation is expected to give more accurate results than lower-order boundary element analysis when nearly singular integrals need to be calculated. Another more important reason for using exact geometrical represen- tation can be found in the following conditions. If the boundary geometry is depicted by using lower order element, the linear element of the outer surface will attach or even pass through the inner boundary if the thickness of the considered structure is very small. Consequently, the actual geometry of considered domain can not be described lively, and thus lowerorder geometry approximation will fail to yield reliable results for such problems. In order to avoid this phenomenon, very fine meshes mush be used in this situation, but this is bound to require more preprocessing and CPU time. Most importantly, a great number of meshes will produce a lot of artificial corners which will lead to the uncontinuity of the tangent derivative of the boundary unknowns. This is fatal to many engineering problems. Obviously, the utilization of exact geometrical representation can be a good choice to avoid this problem. In view of these reasons, this paper will give an efficient strategy to treat thin body problems with circular and elliptic boundaries.

This paper is an extension of our previous work [25] where a new nonlinear transformation method was proposed and applied to treat the thin body effect occurring in 2D potential problems. Herein, we derive a general BEM-based approach to treat thin body problems in $2 \mathrm{D}$ elastostatics. The BEMbased approach proposed in this paper is constructed using a combination of the regularized indirect BIEs and a general nonlinear transformation which can remove or damp out the nearly singular properties of the integral kernels. It is shown that this combined BIE formulation can provide stable results for the analysis of thin body problems. For the test problems studied, very promising results are obtained when the thickness to length ratio of the coatings is in the orders between $1.0 \mathrm{E}-1$ and $1.0 \mathrm{E}-9$, which is sufficient for modeling most thin coated cutting tools in the micro- or nano- scales. In conclusion, the seeming difficult task of determining the physical quantities in thin structures can be dealt with effectively and efficiently.

\section{NON-SINGULAR BOUNDARY INTEGRAL EQUATIONS (BIES)}

It is well known that the domain variables can be computed by integral equations only after all the boundary quantities have been obtained, and the accuracy of boundary quantities directly affects the validity of the interior quantities. However, when calculating the boundary quantities, we have to deal with the singular boundary integrals, and using the regularized boundary integral equations (BIEs).

In this paper, we always assume that $\Omega$ is a bounded domain in $R^{2} ; \Omega^{c}$ is its open complement, and $\Gamma$ denotes the boundary; $\boldsymbol{t}(\boldsymbol{x})$ and $\boldsymbol{n}(\boldsymbol{x})$ (or $\boldsymbol{t}$ and $\boldsymbol{n}$ ) are the unit tangent and outward normal vectors of $\Gamma$ to the domain $\Omega$ at the point $\boldsymbol{x}$, respectively. For $2 \mathrm{D}$ elastic problems, the non-singular BIEs with indirect variables are given in Ref. [29]. Regardless of to the rigid body displacement and the body forces, the non-singular BIEs on $\Omega^{c}$ can be expressed as 


$$
u_{i}(\boldsymbol{y})=\int_{\Gamma} \phi_{k}(\boldsymbol{x}) u_{i k}^{*}(\boldsymbol{y}, \boldsymbol{x}) d \Gamma, \boldsymbol{y} \in \Gamma
$$

$$
\begin{aligned}
\nabla u_{i}(\boldsymbol{y})= & \int_{\Gamma}\left[\phi_{k}(\boldsymbol{x})-\phi_{k}(\boldsymbol{y})\right] \nabla u_{i k}^{*}(\boldsymbol{y}, \boldsymbol{x}) d \Gamma-\phi_{k}(\boldsymbol{y}) \\
& \left\{\int_{\Gamma}[\boldsymbol{t}(\boldsymbol{x})-\boldsymbol{t}(\boldsymbol{y})] \frac{\partial u_{i k}^{*}(\boldsymbol{y}, \boldsymbol{x})}{\partial \boldsymbol{t}} d \Gamma+\int_{\Gamma}[\boldsymbol{n}(\boldsymbol{x})-\boldsymbol{n}(\boldsymbol{y})]\right. \\
& \frac{\partial u_{i k}^{*}(\boldsymbol{y}, \boldsymbol{x})}{\partial \boldsymbol{n}} d \Gamma+\frac{k_{0}}{G} \boldsymbol{n}(\boldsymbol{y})\left(\int_{\Gamma}\left[n_{k}(\boldsymbol{x})-n_{k}(\boldsymbol{y})\right]\right. \\
& \frac{\partial \ln r}{\partial x_{i}} d \Gamma+n_{k}(\boldsymbol{y}) \int_{\Gamma}\left[t_{i}(\boldsymbol{x})-t_{i}(\boldsymbol{y})\right] \frac{\partial \ln r}{\partial \boldsymbol{t}} d \Gamma \\
& \left.\left.+n_{k}(\boldsymbol{y}) \int_{\Gamma}\left[n_{i}(\boldsymbol{x})-n_{i}(\boldsymbol{y})\right] \frac{\partial \ln r}{\partial \boldsymbol{n}} d \Gamma\right)\right\}, \boldsymbol{y} \in \Gamma
\end{aligned}
$$

For the domain $\Omega$, the non-singular BIEs are given as

$$
\begin{gathered}
u_{i}(\boldsymbol{y})=\int_{\Gamma} \phi_{k}(\boldsymbol{x}) u_{i k}^{*}(\boldsymbol{x}, \boldsymbol{y}) d \Gamma, \boldsymbol{y} \in \Gamma \\
\nabla u_{i}(\boldsymbol{y})=\phi_{k}(\boldsymbol{y}) \mathbf{n}(\boldsymbol{y}) \frac{1}{G}\left[\delta_{i k}-\frac{n_{k}(\boldsymbol{y}) n_{i}(\boldsymbol{y})}{2(1-v)}\right]+\int_{\Gamma}\left[\phi_{k}(\boldsymbol{x})\right. \\
\left.-\phi_{k}(\boldsymbol{y})\right] \nabla u_{i k}^{*}(\boldsymbol{y}, \boldsymbol{x}) d \Gamma-\phi_{k}(\boldsymbol{y})\left\{\int_{\Gamma}[\boldsymbol{t}(\mathbf{x})-\boldsymbol{t}(\mathbf{y})]\right. \\
\frac{\partial u_{i k}^{*}(\boldsymbol{y}, \boldsymbol{x})}{\partial \boldsymbol{t}} d \Gamma+\int_{\Gamma}[\boldsymbol{n}(\boldsymbol{x})-\boldsymbol{n}(\boldsymbol{y})] \frac{\partial u_{i k}^{*}(\boldsymbol{y}, \boldsymbol{x})}{\partial \mathbf{n}} d \Gamma \\
+\frac{k_{0}}{G} \boldsymbol{n}(\boldsymbol{y})\left(\int_{\Gamma}\left[n_{k}(\boldsymbol{x})-n_{k}(\boldsymbol{y})\right] \frac{\partial \ln r}{\partial x_{i}} d \Gamma+n_{k}(\boldsymbol{y})\right. \\
\int_{\Gamma}\left[t_{i}(\boldsymbol{x})-t_{i}(\boldsymbol{y})\right] \frac{\partial \ln r}{\partial \boldsymbol{t}} d \Gamma+n_{k}(\boldsymbol{y}) \\
\left.\left.\int_{\Gamma}\left[n_{i}(\boldsymbol{x})-n_{i}(\boldsymbol{y})\right] \frac{\partial \ln r}{\partial \boldsymbol{n}} d \Gamma\right)\right\}, \boldsymbol{y} \in \Gamma
\end{gathered}
$$

For the internal point $\boldsymbol{y}$, the integral equations can be written as

$$
\begin{gathered}
u_{i}(\boldsymbol{y})=\int_{\Gamma} \phi_{k}(\boldsymbol{x}) u_{i k}^{*}(\boldsymbol{y}, \boldsymbol{x}) d \Gamma, \boldsymbol{y} \in \hat{\Omega} \\
\nabla u_{i}(\boldsymbol{y})=\int_{\Gamma} \phi_{k}(\boldsymbol{x}) \nabla u_{i k}^{*}(\boldsymbol{y}, \boldsymbol{x}) d \Gamma, \boldsymbol{y} \in \hat{\Omega}
\end{gathered}
$$

In Eqs. (1) (6), $i, k=1,2 ; k_{0}=1 / 4 \pi(1-v) ; G$ is the shear modulus; $\phi_{k}(\boldsymbol{x})$ is the density function to be determined; $u_{i k}^{*}(\boldsymbol{y}$, $\boldsymbol{x})$ denotes the Kelvin fundamental solution. In Eqs. (5) and (6) $\hat{\Omega}=\Omega$ or $\Omega^{c}$.

Note that, the Gaussian quadrature is directly used to calculate the integrals in discretized equations in the CBEM. However, if the domain of a considered problem is thin, some boundaries will be very close to each other. Thus, the distance $r$ between some boundary nodes and boundary integral elements probably approaches zero. This causes the integrals in discretized Eqs. (1) (4) nearly singular, and the results of the Gaussian quadrature become invalid. Therefore, the density functions cannot be calculated accurately, needless to say, to calculate the physical quantities at interior points. Moreover, almost all the interior points of thin bodies are very close to the integral elements. Thus, there also exist nearly singular integrals in Eqs. (5) and (6). These nearly singular integrals can be expressed as

$$
\left\{\begin{array}{l}
I_{1}=\int_{\Gamma_{e}} \psi(\boldsymbol{x}) \ln r^{2} d \Gamma \\
I_{2}=\int_{\Gamma_{e}} \psi(\boldsymbol{x}) \frac{1}{r^{2 \alpha}} d \Gamma
\end{array}\right.
$$

where $\alpha>0, \psi(\boldsymbol{x})$ is a well-behaved function including the Jacobian, the shape functions, and a finite sum of polynomials divided by $r^{n}$. Under such a circumstance, either a very fine mesh with massive integration points or a special integration technique needs to be adopted. In the last two decades, numerous research works have been published on this subject in the BEM literature. Most of the works have been focused on the numerical approaches, such as subdivisions of the element of integration, adaptive integration schemes, exact integration methods and so on. However, most of these earlier methods are either inefficient or can not provide accurate results when the thickness of the thin structure is smaller than 1.0E-6. In this work, a very efficient transformation method is proposed to avoid the integration difficulty for thin coatings with the thickness to length ratio in micro- or nano- scales.

\section{THE APPROXIMATION OF GEOMETRY BOUNDARIES}

The quintessence of the BEM is to discretize the boundary into a finite number of segments, not necessarily equal, which are called boundary elements. Two approximations are made over each of these elements. One is about the geometry of the boundary, while the other has to do with the variation of the unknown boundary quantity over the element.

\section{Linear Element Approximation}

In this section, the geometry segment is modeled by a continuous linear element.

Assuming $\boldsymbol{x}^{1}=\left(x_{1}^{1}, x_{2}^{1}\right), \boldsymbol{x}^{2}=\left(x_{1}^{2}, x_{2}^{2}\right)$ are the two extreme points of the linear element $\Gamma_{j}$, then the element $\Gamma_{j}$ can be expressed as

$$
x_{k}(\xi)=N_{1}(\xi) x_{k}^{1}+N_{2}(\xi) x_{k}^{2}, \xi \in[-1,1], k=1,2
$$

where $N_{1}(\xi)=(1-\xi) / 2, N_{2}(\xi)=(1+\xi) / 2$.

Letting $s_{i}=x_{i}^{2}-x_{i}^{1}, w_{i}=y_{i}-\left(x_{i}^{2}-x_{i}^{1}\right) / 2$, one has 


$$
\begin{gathered}
r_{, i}=\frac{r_{i}}{r}=\frac{y_{i}-x_{i}}{r}=\frac{s_{i} \xi / 2+w_{i}}{r} \\
r^{2}=|\boldsymbol{x}-\boldsymbol{y}|^{2}=r_{i} r_{i}=A \xi^{2}+B \xi+E=L^{2}\left[(\xi-\eta)^{2}+d^{2}\right]
\end{gathered}
$$

where $A=s_{i} s_{i} / 4, B=s_{i} w_{i}, E=w_{i} w_{i}, \eta \eta=-B / 2 \mathrm{~A}, L=\sqrt{A}, d=$ $\sqrt{4 A E-B^{2}} / 2 A$.

With the aid of the Eq. (10), the nearly singular integrals in Eq. (7) can be rewritten as

$$
\left\{\begin{array}{l}
I_{1}=\left\{\int_{-1}^{\eta}+\int_{\eta}^{1}\right\} g(\xi) \ln \left[(\xi-\eta)^{2}+d^{2}\right] d \xi+\ln L^{2} \int_{-1}^{1} g(\xi) d \xi \\
I_{2}=\left\{\int_{-1}^{\eta}+\int_{\eta}^{1}\right\} \frac{g(\xi)}{L^{2 \alpha}\left[(\xi-\eta)^{2}+d^{2}\right]^{\alpha}} d \xi
\end{array}\right.
$$

where $g(\cdot)$ is a regular function that consists of shape function and Jacobian.

\section{2. "Arc Element" Approximation}

It is well-known that the geometries of many problems of practical interest are created from circular or elliptic arcs. Arc boundary elements can represent circular and elliptic boundaries exactly, and consequently, errors caused by geometries using polynomial shape functions which can be removed by using exact geometrical representations. Therefore, the exact geometrical representation is expected to give more accurate results than lower-order or even high-order boundary element analysis when nearly singular integrals need to be calculated.

An exact geometrical representation, termed "arc element", for circular and elliptic boundaries has been proposed by Zhang [29] in 2004. Consider a circular are element of radius of curvature $R$ with its centre of curvature located at $\left(x_{1}^{\prime}, x_{2}^{\prime}\right)$. Suppose $\left(R, \theta_{1}\right),\left(R, \theta_{2}\right)$ are the coordinates of the two extreme points of the arc element $\Gamma_{j}$, respectively. Then the exact boundary elements can be expressed as:

$$
\left\{\begin{array}{l}
x_{1}(\xi)=x_{1}^{\prime}+R \cos \theta \\
x_{2}(\xi)=x_{2}^{\prime}+R \sin \theta
\end{array}\right.
$$

where $\theta=\frac{1-\xi}{2} \theta_{1}+\frac{1+\xi}{2} \theta_{2},(-1 \leq \xi \leq 1)$.

The Jacobian of transformation from arc element to intrinsic coordinate $\xi$ reduces to:

$$
J(\xi)=R \frac{\left|\theta_{1}-\theta_{2}\right|}{2}
$$

For the interior point $y=\left(R_{0} \cos \theta_{0}, R_{0} \sin \theta_{0}\right)$, we can suppose $\theta_{1}<\theta_{0}<\theta_{2}$ and $\left(x_{1}^{\prime}, x_{2}^{\prime}\right)=(0,0)$. Then

$$
\theta_{0}=\frac{1-\eta}{2} \theta_{1}+\frac{1+\eta}{2} \theta_{2}(-1<\eta<1)
$$

Using the procedure described above, the distance between the source point and the field point can be expressed as:

$$
r^{2}=|\boldsymbol{x}-\boldsymbol{y}|^{2}=4 R R_{0}\left\{\sin ^{2} \gamma+d^{2}\right\}
$$

where $\gamma=\beta(\xi-\eta), \beta=\left(\theta_{2}-\theta_{1}\right) / 4, d=\frac{R-R_{0}}{4 \sqrt{R R_{0}}}$.

Substituting $r^{2}$ into Eq. (7), then the integrals $I_{1}$ and $I_{2}$ can be divided into two parts at point $\eta$ as follows

$$
\left\{\begin{array}{l}
I_{1}=\left\{\int_{-1}^{\eta}+\int_{\eta}^{1}\right\} g(\xi) \ln \left(\sin ^{2} \gamma+d^{2}\right) d \xi+\ln L^{2} \int_{-1}^{1} g(\xi) d \xi \\
I_{2}=\frac{1}{L^{2 \alpha}}\left\{\int_{-1}^{\eta}+\int_{\eta}^{1}\right\} \frac{g(\xi)}{\left(\sin ^{2} \gamma+d^{2}\right)^{\alpha}} d \xi
\end{array}\right.
$$

where $L=2 \sqrt{R R_{0}}$ and $g(\cdot)$ is a regular function that consists of shape function, the Jacobian and a finite sum of polynomials divided by $r^{n}$.

\section{THE TRANSFORMATION FOR NEARLY SINGULAR INTEGRAL}

In Eqs. (11) and (15), if $d$ is very small, the above integrals would present various orders of near singularity. The key to achieving high accuracy is to find a method to calculate these integrals accurately for a small value of $d$.

The integrals $I_{1}$ and $I_{2}$ in Eqs. (11) and (15) can be reduced to the following integrals by simple deduction

$$
\left\{\begin{array}{l}
T_{1}=\int_{0}^{A} g(x) \ln \left(x^{2}+d^{2}\right) d x \\
T_{2}=\int_{0}^{A} \frac{g(x)}{\left(x^{2}+d^{2}\right)^{\alpha}} d x
\end{array}\right.
$$

Introducing the following nonlinear transformation

$$
x=d\left(e^{k(1+t)}-1\right), t \in[-1,1]
$$

where $k=\ln \sqrt{1+A / d}$.

Substituting (17) into (16), then the integrals $I_{1}$ and $I_{2}$ can be rewritten as follows:

$$
\left\{\begin{aligned}
T_{1}= & d k \int_{-1}^{1} g(t) \ln d^{2} e^{k(1+t)} d t \\
& +d k \int_{-1}^{1} g(t) \ln \left[\left(e^{k(1+t)}-1\right)^{2}+1\right] e^{k(1+t)} d t \\
T_{2}= & d^{1-2 \alpha} k \int_{-1}^{1} \frac{g(t) e^{k(1+t)}}{\left[\left(e^{k(1+t)}-1\right)^{2}+1\right]^{\alpha}} d t
\end{aligned}\right.
$$

We can observe that $\left(e^{k(1+t)}-1\right)^{2}+1 \geq 1$. Thus, the inte- 


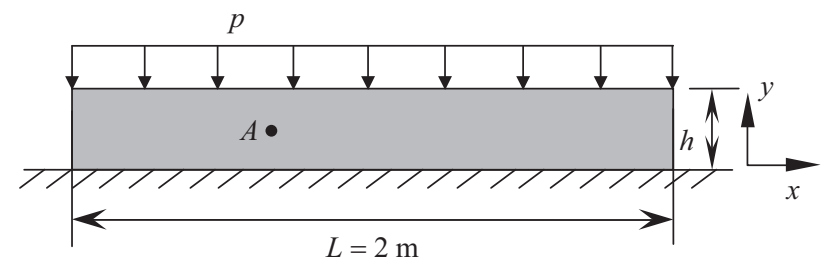

Fig. 1. A thin plate under constant pressure $p$ (Shear modulus $\mu=8.0 \times$ $10^{10}$, Poisson's ratio $v=0.2$ ).

grand is fully regular even if the value of $d$ is very small. By following the procedures described above, the near singularity in the BIEs has been fully regularized. The final integral formulations are obtained as shown in Eq. (18), and be computed straightforwardly by using the standard Gaussian quadrature.

\section{NUMERICAL EXAMPLES}

To verify the method developed above, two simple test problems are studied in which BEM solutions are compared with the exact solutions.

\section{Test Problem 1: A Thin Plate}

First, a thin plate under external pressure $p$ shown in Fig. 1 is studied. We assume the length of the plate in $z$ direction is large so that this problem can be simplified as a plane strain problem. The length $L$ of the plate in $x$ direction is constant in this study, while the thickness $h$ changes from $L$ to $10^{-9} L$. Note that the thickness is changing from macro-scale to microscale, and eventually to nano-scale. On the boundary $y=0$, displacement components in both $x$ and $y$ directions are constrained.

The finite element analysis of this problem was attempted in Ref. [14], but it was soon found out that the number of the 2D finite element was so large that the task quickly exceed the capacity of the computer used. In the BEM model, the boundary of the plate is discretized with only 20 linear boundary elements, 16 elements with length $L=2 \mathrm{~m}$, and 4 other elements with thickness $h$.

When the thickness $h$ ranges from $1.0 \mathrm{E}-1$ to $1.0 \mathrm{E}-10$, the results of the normal stresses $\sigma_{x x}$ and $\sigma_{y y}$ at interior point $A(0.8$, $h / 2$ are shown in Fig. 2 and Fig. 3, respectively. It is obvious that the results calculated by using the CBEM deteriorate quickly as the thickness less than $1.0 \mathrm{E}-2$. In contrast, the results calculated by using the proposed method are very consistent with the exact solutions even for the thickness as small as $1.0 \mathrm{E}-10$. This proves that the developed transformation work in the BEM procedure is effective. For $h=1.0 \mathrm{E}-9$, the stress results at the interior points on the line $x=1.8$ are listed in Table 1 and Table 2. These results calculated by the present BEM are all in good agreement with the exact solutions with the largest relative error less than 2.0E-4 (\%). However, the CBEM are invalid to calculate both the radial and tangential stresses at these points.

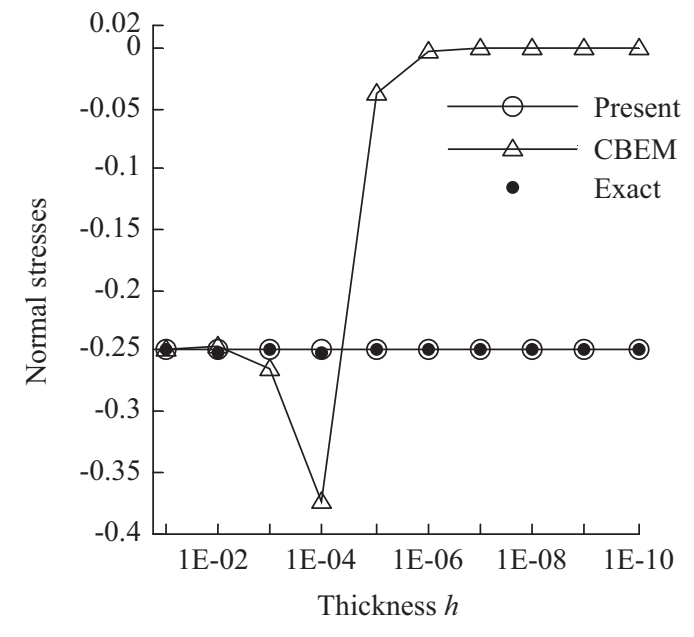

Fig. 2. Normal stresses $\sigma_{x x}$ at the interior point $A$ of Fig. 1.

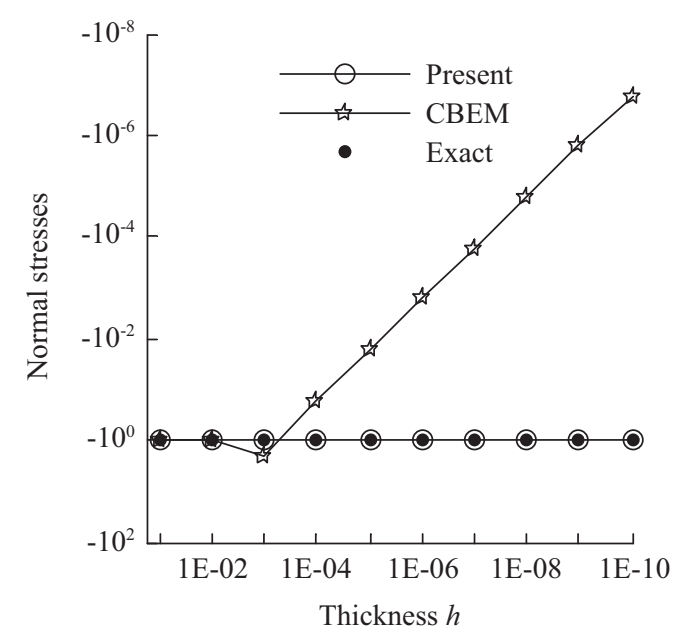

Fig. 3. Normal stresses $\sigma_{y y}$ at the interior point $A$ of Fig. 1.

The example problem studied here is a very simple one in thin structures. The purpose of using this example is to verify the correctness of the present transformation method in dealing with nearly singular integrals.

\section{Test Problem 2: Thin Coating on a Shaft}

In this section, the method developed in this paper will be used to solve a problem of a shaft with a thin coating [14], as shown in Fig. 4. The shaft and coating have outer radii $r_{a}$ and $r_{b}$ respectively, with their centre of curvature located at the point $o(0,0)$. In this example, the coated system is loaded by a uniform pressure $p=1$, and the shaft is considered to be rigid when compared to the coating, so the boundary conditions are $u_{x}=u_{y}=0$ for all nodes at the shaft/coating interface. In this example, $\delta=\left(r_{b}-r_{a}\right) / r_{a}$ is defined as the thickness to length ratio. As $r_{a}$ is held constant at 1 , the ratio reduces as $r_{b}$ decreases.

There are totally 20 discontinuous "arc elements" divided along the shaft and coating surfaces, regardless of the thick- 
Table 1. Normal stresses $\sigma_{x x}$ at the interior points on the line $x=1.8$, where $h=1.0 \mathrm{E}-9$.

\begin{tabular}{cccccc}
\hline$y$ & Exact & CBEM & Relative error (\%) & Present & Relative error (\%) \\
\hline $1.0 \mathrm{E}-10$ & -0.25 & $-0.2633707 \mathrm{E}-06$ & $0.9999989 \mathrm{E}+02$ & $-0.2500004 \mathrm{E}+00$ & $-0.1443958 \mathrm{E}-03$ \\
$2.0 \mathrm{E}-10$ & -0.25 & $-0.2633707 \mathrm{E}-06$ & $0.9999989 \mathrm{E}+02$ & $-0.2500001 \mathrm{E}+00$ & $-0.5765732 \mathrm{E}-04$ \\
$3.0 \mathrm{E}-10$ & -0.25 & $-0.2633707 \mathrm{E}-06$ & $0.9999989 \mathrm{E}+02$ & $-0.2500002 \mathrm{E}+00$ & $-0.6570008 \mathrm{E}-04$ \\
$4.0 \mathrm{E}-10$ & -0.25 & $-0.2633707 \mathrm{E}-06$ & $0.9999989 \mathrm{E}+02$ & $-0.2500001 \mathrm{E}+00$ & $-0.4155786 \mathrm{E}-04$ \\
$5.0 \mathrm{E}-10$ & -0.25 & $-0.2633707 \mathrm{E}-06$ & $0.9999989 \mathrm{E}+02$ & $-0.2500001 \mathrm{E}+00$ & $-0.5236237 \mathrm{E}-04$ \\
$6.0 \mathrm{E}-10$ & -0.25 & $-0.2633707 \mathrm{E}-06$ & $0.9999989 \mathrm{E}+02$ & $-0.2500001 \mathrm{E}+00$ & $-0.4155786 \mathrm{E}-04$ \\
$7.0 \mathrm{E}-10$ & -0.25 & $-0.2633707 \mathrm{E}-06$ & $0.9999989 \mathrm{E}+02$ & $-0.2500002 \mathrm{E}+00$ & $-0.6570008 \mathrm{E}-04$ \\
$8.0 \mathrm{E}-10$ & -0.25 & $-0.2633707 \mathrm{E}-06$ & $0.9999989 \mathrm{E}+02$ & $-0.2500001 \mathrm{E}+00$ & $-0.5765732 \mathrm{E}-04$ \\
$9.0 \mathrm{E}-10$ & -0.25 & $-0.2633707 \mathrm{E}-06$ & $0.9999989 \mathrm{E}+02$ & $-0.2500004 \mathrm{E}+00$ & $-0.1443958 \mathrm{E}-03$ \\
\hline
\end{tabular}

Table 2. Normal stresses $\sigma_{y y}$ at the interior points on the line $x=1.8$, where $h=1.0 \mathrm{E}-9$.

\begin{tabular}{cccccc}
\hline$y$ & Exact & CBEM & Relative error (\%) & Present & Relative error (\%) \\
\hline $1.0 \mathrm{E}-10$ & -1.0 & $-0.1174473 \mathrm{E}-06$ & $0.9999999 \mathrm{E}+02$ & $-0.9999998 \mathrm{E}+00$ & $0.1649320 \mathrm{E}-04$ \\
$2.0 \mathrm{E}-10$ & -1.0 & $-0.1174473 \mathrm{E}-06$ & $0.9999999 \mathrm{E}+02$ & $-0.9999999 \mathrm{E}+00$ & $0.1442842 \mathrm{E}-04$ \\
$3.0 \mathrm{E}-10$ & -1.0 & $-0.1174473 \mathrm{E}-06$ & $0.9999999 \mathrm{E}+02$ & $-0.9999999 \mathrm{E}+00$ & $0.1284025 \mathrm{E}-04$ \\
$4.0 \mathrm{E}-10$ & -1.0 & $-0.1174473 \mathrm{E}-06$ & $0.9999999 \mathrm{E}+02$ & $-0.9999999 \mathrm{E}+00$ & $0.1196222 \mathrm{E}-04$ \\
$5.0 \mathrm{E}-10$ & -1.0 & $-0.1174473 \mathrm{E}-06$ & $0.9999999 \mathrm{E}+02$ & $-0.9999999 \mathrm{E}+00$ & $0.1168348 \mathrm{E}-04$ \\
$6.0 \mathrm{E}-10$ & -1.0 & $-0.1174473 \mathrm{E}-06$ & $0.9999999 \mathrm{E}+02$ & $-0.9999999 \mathrm{E}+00$ & $0.1196222 \mathrm{E}-04$ \\
$7.0 \mathrm{E}-10$ & -1.0 & $-0.1174473 \mathrm{E}-06$ & $0.9999999 \mathrm{E}+02$ & $-0.9999999 \mathrm{E}+00$ & $0.1284025 \mathrm{E}-04$ \\
$8.0 \mathrm{E}-10$ & -1.0 & $-0.1174473 \mathrm{E}-06$ & $0.9999999 \mathrm{E}+02$ & $-0.9999999 \mathrm{E}+00$ & $0.1442842 \mathrm{E}-04$ \\
$9.0 \mathrm{E}-10$ & -1.0 & $-0.1174473 \mathrm{E}-06$ & $0.9999999 \mathrm{E}+02$ & $-0.9999998 \mathrm{E}+00$ & $0.1649320 \mathrm{E}-04$ \\
\hline
\end{tabular}

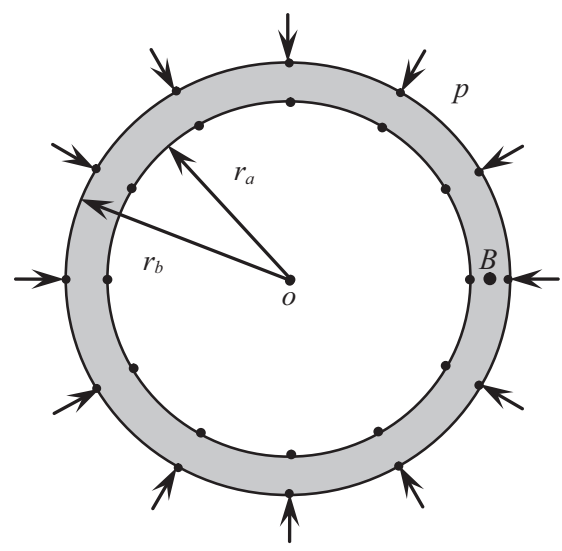

Fig. 4. A shaft with a thin uniform coating.

ness of the structure. The elastic shear modulus is $G=8.0 \times$ $10^{10} \mathrm{~Pa}$, Poisson's ratio is $v=0.2$.

In 1998, Luo et al. [14] have handled this coating system. However, in their work only boundary unknown radial stresses $\sigma_{r}$ are computed. The physical quantities at interior points need further investigation.

For different thickness-to-length ratios, the results of the radial and tangential stresses at interior point $B\left(\left(r_{a}+r_{b}\right) / 2,0\right)$ are listed in Table 3. For $\delta=1.0 \mathrm{E}-9$, the results of tangential stresses on the line $y=0$ are listed in Table 4 . Both the CBEM and the proposed method are employed for the purpose of comparison.
It is obvious that the results calculated by using the proposed method, shown in Table 3, are very consistent with the exact solutions even for the thickness to length ratio as small as $1.0 \mathrm{E}-10$. We can see from Table 4 that the results of tangential stresses calculated by using the proposed method are very consistent with the exact solutions, with the largest relative error less than $0.2 \%$, even when the thickness-to-length ratio as small as $1.0 \mathrm{E}-9$.

For $\delta=1.0 \mathrm{E}-9$, convergence curves of the computed tangential stresses at interior points $B$ by using the presented method are shown in Fig. 5, which we can observe that the convergence speeds are still fast even when the thicknessto-length ratio is as small as 1.0E - 9. In Fig. 5, only the errors of the present method are given since the errors of the CBEM are relatively too large.

\section{CONCLUSION}

In this paper, a BEM-based approach is presented and applied to deal with 2-D elastic problems of thin bodies. Using the transformation technique demonstrated in this paper, the seemingly difficult task of evaluating the nearly singular integrals in the BIE for 2-D thin structures can be dealt with effectively and efficiently. Two numerical examples of elastic thin-walled structures with thickness-to-length ratios ranging between $1.0 \mathrm{E}-1$ and $1.0 \mathrm{E}-9$, which is sufficient for modeling most thin structures in industrial applications, are presented to test the proposed method. In conclusion, the 
Table 3. Radial and Tangential stresses at the interior point $B$ of Fig. 4.

\begin{tabular}{ccc|cc}
\hline \multirow{2}{*}{$\delta$} & \multicolumn{2}{c|}{ Radial stresses $\sigma_{r}$} & \multicolumn{2}{c}{ Tangential stresses $\sigma_{\theta}$} \\
\cline { 2 - 5 } & Exact & Present & Exact & $-0.3046613 \mathrm{E}+00$ \\
$1.0 \mathrm{E}-01$ & $-0.1032322 \mathrm{E}+01$ & $-0.1032346 \mathrm{E}+01$ & $-0.3046943 \mathrm{E}+00$ & $-0.2557357 \mathrm{E}+00$ \\
$1.0 \mathrm{E}-02$ & $-0.1003694 \mathrm{E}+01$ & $-0.1003546 \mathrm{E}+01$ & $-0.2556108 \mathrm{E}+00$ & $-0.2506734 \mathrm{E}+00$ \\
$1.0 \mathrm{E}-03$ & $-0.1000374 \mathrm{E}+01$ & $-0.1000387 \mathrm{E}+01$ & $-0.2505624 \mathrm{E}+00$ & $-0.2502721 \mathrm{E}+00$ \\
$1.0 \mathrm{E}-04$ & $-0.1000037 \mathrm{E}+01$ & $-0.1000038 \mathrm{E}+01$ & $-0.2500562 \mathrm{E}+00$ & $-0.2496582 \mathrm{E}+00$ \\
$1.0 \mathrm{E}-05$ & $-0.1000004 \mathrm{E}+01$ & $-0.1000004 \mathrm{E}+01$ & $-0.2500056 \mathrm{E}+00$ & $-0.2495967 \mathrm{E}+00$ \\
$1.0 \mathrm{E}-06$ & $-0.1000000 \mathrm{E}+01$ & $-0.1000000 \mathrm{E}+01$ & $-0.2500006 \mathrm{E}+00$ & $-0.2495906 \mathrm{E}+00$ \\
$1.0 \mathrm{E}-07$ & $-0.1000000 \mathrm{E}+01$ & $-0.1000000 \mathrm{E}+01$ & $-0.2500001 \mathrm{E}+00$ & $-0.2495901 \mathrm{E}+00$ \\
$1.0 \mathrm{E}-08$ & $-0.1000000 \mathrm{E}+01$ & $-0.9999999 \mathrm{E}+00$ & $-0.2500000 \mathrm{E}+00$ & $-0.2495896 \mathrm{E}+00$ \\
$1.0 \mathrm{E}-09$ & $-0.1000000 \mathrm{E}+01$ & $-0.1000000 \mathrm{E}+01$ & $-0.2500000 \mathrm{E}+00$ & $-0.2495898 \mathrm{E}+00$ \\
$1.0 \mathrm{E}-10$ & $-0.1000000 \mathrm{E}+01$ & $-0.9999984 \mathrm{E}+00$ & $-0.2500000 \mathrm{E}+00$ & \\
\hline
\end{tabular}

Table 4. Tangential stress $\sigma_{\theta}$ at the interior point on the line $y=0$, where $\delta=1.0 \mathrm{E}-9$.

\begin{tabular}{cccccc}
\hline$x$ & Exact & CBEM & Relative error (\%) & Present & Relative error $(\%)$ \\
\hline 1.0000000001 & -0.25 & $0.2015379 \mathrm{E}+01$ & $0.9061517 \mathrm{E}+03$ & $-0.2495897 \mathrm{E}+00$ & $0.1641369 \mathrm{E}+00$ \\
1.0000000002 & -0.25 & $0.2015378 \mathrm{E}+01$ & $0.9061514 \mathrm{E}+03$ & $-0.2495895 \mathrm{E}+00$ & $0.1641839 \mathrm{E}+00$ \\
1.0000000003 & -0.25 & $0.2015378 \mathrm{E}+01$ & $0.9061510 \mathrm{E}+03$ & $-0.2495895 \mathrm{E}+00$ & $0.1641885 \mathrm{E}+00$ \\
1.0000000004 & -0.25 & $0.2015377 \mathrm{E}+01$ & $0.9061507 \mathrm{E}+03$ & $-0.2495896 \mathrm{E}+00$ & $0.1641685 \mathrm{E}+00$ \\
1.0000000006 & -0.25 & $0.2015375 \mathrm{E}+01$ & $0.9061500 \mathrm{E}+03$ & $-0.2495896 \mathrm{E}+00$ & $0.1641754 \mathrm{E}+00$ \\
1.0000000007 & -0.25 & $0.2015374 \mathrm{E}+01$ & $0.9061497 \mathrm{E}+03$ & $-0.2495896 \mathrm{E}+00$ & $0.1641706 \mathrm{E}+00$ \\
1.0000000008 & -0.25 & $0.2015373 \mathrm{E}+01$ & $0.9061494 \mathrm{E}+03$ & $-0.2495895 \mathrm{E}+00$ & $0.1641929 \mathrm{E}+00$ \\
1.0000000009 & -0.25 & $0.2015373 \mathrm{E}+01$ & $0.9061491 \mathrm{E}+03$ & $-0.2495895 \mathrm{E}+00$ & $0.1641836 \mathrm{E}+00$ \\
\hline
\end{tabular}

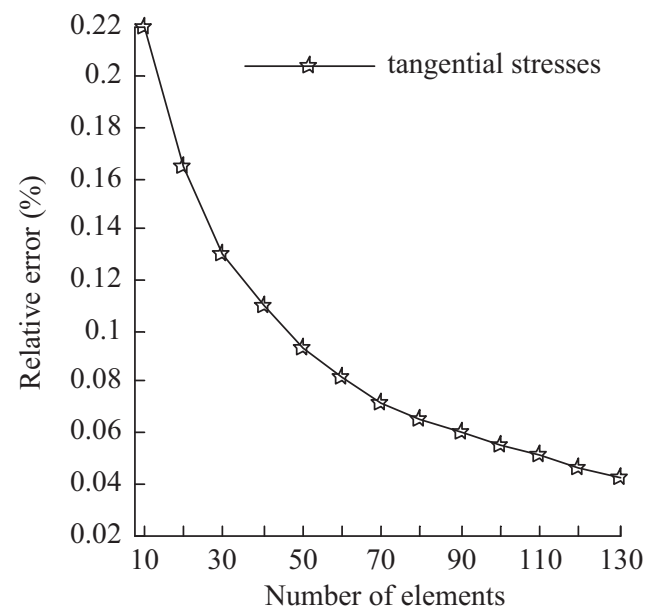

Fig. 5. Convergence curves of $\sigma_{\theta}$ at the interior point $B$ of Fig. 4.

thin-body problem has been overcome successfully by using the proposed strategy, which indicates that BEM is especially accurate and efficient for numerical analysis of thin boy problems. The developed method for analyzing 2-D thin structures can be extended to model layered coatings, thin films or other layered structures, and some work along this line for thin structures will be discussed later.
The research is supported by the National Natural Science Foundation of China (10571110), the Opening Fund of the State Key Laboratory of Structural Analysis for Industrial Equipment (GZ1017) and the National Natural Science Foundation of Shangdong Province of China (ZR2010AZ003).

\section{REFERENCES}

1. Brebbia, C. A., Tells, J. C. F., and Wrobel, L. C., Boundary Element Techniques, Springer-Verlag, Berlin, Germany (1984).

2. Chen, J. T., Chen, P. Y., and Chia, T. C., "Surface motion of multiple alluvial valleys for incident plane $\mathrm{SH}$-waves by using a semi-analytical approach," Soil Dynamics and Earthquake Engineering, Vol. 28, No. 3, pp. 58-72 (2008).

3. Chen, J. T. and Hong, H. K., "Review of dual boundary element methods with emphasis on hypersingular integrals and divergent series," Applied Mechanics Reviews, Vol. 52, No. 1, pp. 17-33 (1999).

4. Chen, J. T. and Shen, W. C., "Degenerate scale for multiply connected laplace problems," Mechanics Research Communications, Vol. 34, No. 1, pp. 69-77 (2007b).

5. Granados, J. J. and Gallego, G., "Regularization of nearly hypersingular integrals in the boundary element method," Engineering Analysis with Boundary Elements, Vol. 25, No. 3, pp. 165-184 (2001).

6. Hong, H.-K. and Chen, J. T., "Derivations of integral equations of elasticity," Journal of Engineering Mechanics, ASCE, Vol. 114, No. 6, pp. 1028-1044 (1988).

7. Hsiao, S. S., Lin, M. C., and Hu, N. C., "Drbem analysis of combined wave refraction and diffraction in the presence of current," Journal of Marine Science and Technology, Vol. 10, No. 1, pp. 54-60 (2002).

8. Huang, Q. and Cruse, T. A., "Some notes on singular integral techniques in boundary element analysis," International Journal for Numerical 
Methods in Engineering, Vol. 36, No. 15, pp. 2643-2659 (1993).

9. Jun, L., Beer, G., and Meek, J. L., "Efficient evaluation of integrals of order using Gauss quadrature," Engineering Analysis, Vol. 2, No. 3, pp. 118-123 (1985).

10. Liu, C. S., "A modified collocation Trefftz method for the inverse Cauchy problem of laplace equation," Engineering Analysis with Boundary Elements, Vol. 32, No. 9, pp. 778-785 (2006).

11. Liu, C. S., Chang, C. W., and Chiang, C. Y., "A regularized integral equation method for the inverse geometry heat conduction problem," International Journal of Heat and Mass Transfer, Vol. 51, No. 21, pp. 5380-5388 (2008).

12. Liu, Y. J., "Analysis of shell-like structures by the boundary element method based on 3-D elasticity: formulation and verification," International Journal for Numerical Methods in Engineering, Vol. 41, No. 3 , pp. 541-558 (1998)

13. Liu, Y. J., "On the simple solution and non-singular nature of the BIE/BEM-a review and some new results," Engineering Analysis with Boundary Elements, Vol. 24, No. 10, pp. $789-795$ (2000).

14. Luo, J. F., Liu, Y. J., and Berger, E. J., "Analysis of two-dimensional thin structures (from micro- to nano-scales) using the boundary element method," Computational Mechanics, Vol. 22, pp. 404-412 (1998).

15. Lutz, E. L., "Exact Gaussian quadrature methods for near-singular integrals in the boundary element method," Engineering Analysis with Boundary Elements, Vol. 9, No. 3, pp. 233-245 (1992).

16. Ma, H. and Kamiya, N., "Distance transformation for the numerical evaluation of near singular boundary integrals with various kernels in boundary element method," Engineering Analysis with Boundary Elements, Vol. 25, No. 4, pp. 329-339 (2002a).

17. Mukerjee, S., Chati, M. K., and Shi, X. L., "Evaluation of nearly singular integrals in boundary element contour and node methods for threedimensional linear elasticity," International Journal of Solids and Structures, Vol. 37, No. 51, pp. 7633-7654 (2000).

18. Niu, Z. R., Wang, X. X., and Zhou, H. L., "A general algorithm for calculating the quantities at interior points close to the boundary by the BEM," Chinese Journal of Theoretical and Applied Mechanics, Vol. 33, No. 2, pp. 275-283 (2001). (in Chinese)

19. Sladek, V. and Sladek, J., Singular Integrals in Boundary Element Methods, Computational Mechanics Publications, Southampton, UK and Boston (1998).

20. Sladek, V., Sladek, J., and Tanaka, M., "Regularization of hypersingula and nearly singular integrals in the potential theory and elasticity," International Journal for Numerical Methods in Engineering, Vol. 36, No. 10, pp. 1609-1628 (1993).

21. Sladek, V., Sladek, J., and Tanaka, M., "Nonsingular BEM formulations for thin-walled structures and elastostatic crack problems," Acta Mechanica, Vol. 99, pp. 173-190 (1993).

22. Sun, H. C., Nonsingular Boundary Element Method, Dalian University of Technology Press (1999). (in Chinese)

23. Tanaka, M., Sladek, V., and Sladek, J., "Regularization techniques applied to BEM," Applied Mechanics Reviews, Vol. 47, No. 10, pp. 457-499 (1994).

24. Wang, W. H., Liou, J. H., Robert, S., and Ben, D., "Machine vibration induced underwater acoustic radiation," Journal of Marine Science and Technology, Vol. 8, No. 1, pp. 30-40 (2000).

25. Zhang, Y. M. and Gu, Y., "An effective method in BEM for potential problems of thin bodies," Journal of Marine Science and Technology, Vol. 18, No. 1, pp. 137 -144 (2010).

26. Zhang, Y. M. and Sun, C. L., "A general algorithm for the numerical evaluation of nearly singular boundary integrals in the equivalent nonsingular BIEs with indirect unknowns," Journal of the Chinese Institute of Engineers, Vol. 31, No. 3, pp. 437-447 (2008).

27. Zhang, Y. M. and Sun, H. C., "Theoretic analysis on virtual boundary element," Chinese Journal of Computational Mechanics, Vol. 17, No. 1, pp. 56-62 (2000). (in Chinese)

28. Zhang, Y. M. and Sun, H. C., "Analytical treatment of boundary integrals in direct boundary element analysis of plane potential elasticity problems," Applied Mathematic and Mechanics, Vol. 6, pp. 664-673 (2001). (in Chinese)

29. Zhang, Y. M., Wen, W. D., Wang, L. M., and Zhao, X. Q., "A kind of new nonsingular boundary integral equations for elastic plane problems," Acta Mech, Vol. 36, No. 3, pp. 311-321 (2004). (in Chinese)

30. Zhou, H. L., Niu, Z. R., Cheng, C. Z., and Guan, Z. W., "Analytical integral algorithm in the BEM for orthotropic potential problems of thin body," Engineering Analysis with Boundary Elements, Vol. 31, pp. 739748 (2007)

31. Zhou, H. L., Niu, Z. R., Cheng, C. Z., and Guan, Z. W., "Analytical integral algorithm applied to boundary layer effect and thin body effect in BEM for anisotropic potential problems," Computers and Structures, Vol. 86, pp. 1656-1671 (2008). 